\title{
Presynaptic inhibition of monosynaptic reflexes in the lower limbs of subjects with upper motoneuron disease
}

\author{
JF ILES, RC ROBERTS* \\ From the Department of Zoology, University of Oxford and the Department of Neurology, Radcliffe \\ Infirmary, ${ }^{*}$ Oxford, UK
}

SUMMARY Presynaptic inhibition of muscle spindle Ia afferents by group I afferents from the same and other muscles has been studied in the lower limbs of subjects with upper motoneuron lesions. The experiments utilised conditioning of soleus test monosynaptic reflexes during controlled voluntary contraction. The protocol was designed to isolate presynaptic inhibition from postsynaptic components. The relation between estimate of inhibition and test reflex amplitude was examined. The subjects showed less inhibition than controls at all levels of voluntary torque investigated $(\leqslant 15 \mathrm{Nm})$. Two thirds had weak inhibition which did not show the decrease during muscle contraction characteristic of controls. The degree of difference from the normal situation correlated with severity of the clinical sign (weakness of voluntary ankle flexion).

In normal human subjects, vibration of the soleus muscle, which strongly excites muscle spindle afferents, inhibits soleus monosynaptic reflexes. This action is generally regarded as resulting from an autogenetic presynaptic inhibition of the soleus muscle spindle Ia afferents which are responsible for the reflex. There have been several reports that vibration has little effect on reflexes in subjects having upper motoneuron lesions and spasticity in the lower limbs. ${ }^{1-4}$ The conclusion drawn from this work is that presynaptic inhibition of Ia afferents by group I afferents is reduced with upper motoneuron lesions. This change could contribute to the production of exaggerated stretch reflexes.

Almost all of these experiments have utilised soleus test monosynaptic reflexes, conditioned by soleus vibration, in subjects at rest. Such experiments are difficult or impossible to interpret quantitatively, for several reasons. First, and most importantly, a change in the action of vibration on monosynaptic reflex amplitude in the diseased state could result from alter-

Present address and address for reprints: Dr RC Roberts, University Department of Clinical Neurology, The National Hospital, Queen Square, London WCIN 3BG, UK.

Received 13 August 1985 and in revised form 28 October 1985. Accepted 2 November 1985 ation of other excitatory or inhibitory pathways to the test motoneurons, either as well as, or instead of changes in presynaptic inhibition. Even experiments in which reflexes are tested just after a period of vibration ${ }^{4}$ could include long lasting postsynaptic effects. In these cases it cannot be excluded that changes in spinal pathways other than those of presynaptic inhibition are involved. Second, vibration of soleus itself could alter the amplitude of the test afferent volley, thus producing quantitatively misleading results. This is particularly likely when the reflex is induced mechanically because vibration is known to increase muscle spindle responses to stretch. Third, presynaptic inhibition has been found to weaken during contraction of the test muscle in normal subjects. ${ }^{5}$ Given that it may be difficult to relax fully the muscles of spastic subjects, ${ }^{67}$ there is a possibility that the reduced inhibition found in them at "rest", could, in fact, result from small unintended contractions. Fourth, many examples of inhibition when examined by reflex testing appear weaker when the test reflex is very large. ${ }^{8}$ Since soleus $\mathrm{H}$ reflexes are known to be enhanced in spasticity, ${ }^{9}$ this factor could lead to an apparent reduction in presynaptic inhibition in those subjects even if transmission of the inhibitory action in the spinal cord was actually unchanged. Fifth, presynaptic inhibition can also be produced by vibration of muscles other than the test 
muscle. ${ }^{5}$ Changes in these inhibitory pathways could contribute to spasticity.

We have reinvestigated presynaptic inhibition in subjects with upper motoneuron lesions using experimental methods designed to avoid some of these difficulties of interpretation and in particular to try to isolate presynaptic inhibition. Subjects were studied both at rest (for comparison with most published work) and also during voluntary soleus contractions at constant torque. Inhibitory action under these conditions should be largely presynaptic. This can be illustrated by the following example. When a conditioning vibration of muscle is applied during voluntary contraction any postsynaptic actions of afferents excited by vibration will lead to a change in the firing of soleus motoneurons and thus of torque. The subject will compensate by a change of effort and thus of descending control, restoring torque to its previous value and at the same time restoring the soleus motoneuron pool to its original state. If the reflex discharge of that soleus motoneuron pool to a monosynaptic input is smaller during vibration a presynaptic mechanism must be active. Since experiments were performed both at rest and during controlled muscle contraction inadequate relaxation could not influence all of the results. Test reflex amplitude was deliberately varied in order to check for influence on the estimates of inhibition. Conditioning stimuli were obtained from several different muscles. A brief account of some of this work has been published. ${ }^{10}$.

\section{Subjects and methods}

A total of 17 subjects with upper motoneuron lesions attending Oxford Hospitals were studied with their informed con- sent. The experimental protocols were approved by the Oxford AHA Sector Ethics Committee and by the patients' physicians.

Subjects with upper motoneuron lesions and spasticity affecting one or both legs were selected, regardless of aetiology. However, since we wished to study inhibition during controlled voluntary contraction, only subjects with some degree of power and voluntary control were appropriate. All were able to walk at least a few steps. The experimental group was thus highly selected. Even so, three subjects proved upon examination to be unsuited to further study. This was due either to weakness, or to vigorous clonus induced by muscle vibration. This left an experimental group of 14 subjects (nine male: five female) whose clinical details are summarised in the table. They are identified by initials here and in the Results section, and by unique symbols in the figures.

Clinical assessment of spasticity (on a scale from 0: normal tone to 4: very severe spasticity ${ }^{11}$ ) and muscle power (MRC scale; 0 : no contraction to 5: normal power) was made before the experiments commenced. The description of clonus $(Y$ : present, $\mathbf{N}$ : absent) refers to clonus following forced ankle dorsiflexion during clinical examination. Some subjects classified as lacking clonus nevertheless developed it in response to muscle vibration.

Subject AS was investigated both before and two weeks after an operation for relief of cord compression. Subjects LI and PB were reinvestigated about 3 years after the original study.

Only one subject was receiving drugs to relieve spasticity during the investigation (PB: diazepam, $2 \mathrm{mg}$ bd on the first occasion; diazepam $2 \mathrm{mg}$ and baclofen $10 \mathrm{mg}$ bd on the second).

Data from a group of twelve normal subjects used in earlier investigations were available for comparison. However, all of these subjects were young adults (age $<38$ years). Since our spastic subjects ranged in age from 29 to 73 years, and there is evidence that presynaptic inhibition varies with age, ${ }^{2}$ we decided to extend the control group's age distribu-

Table Clinical details of spastic subjects

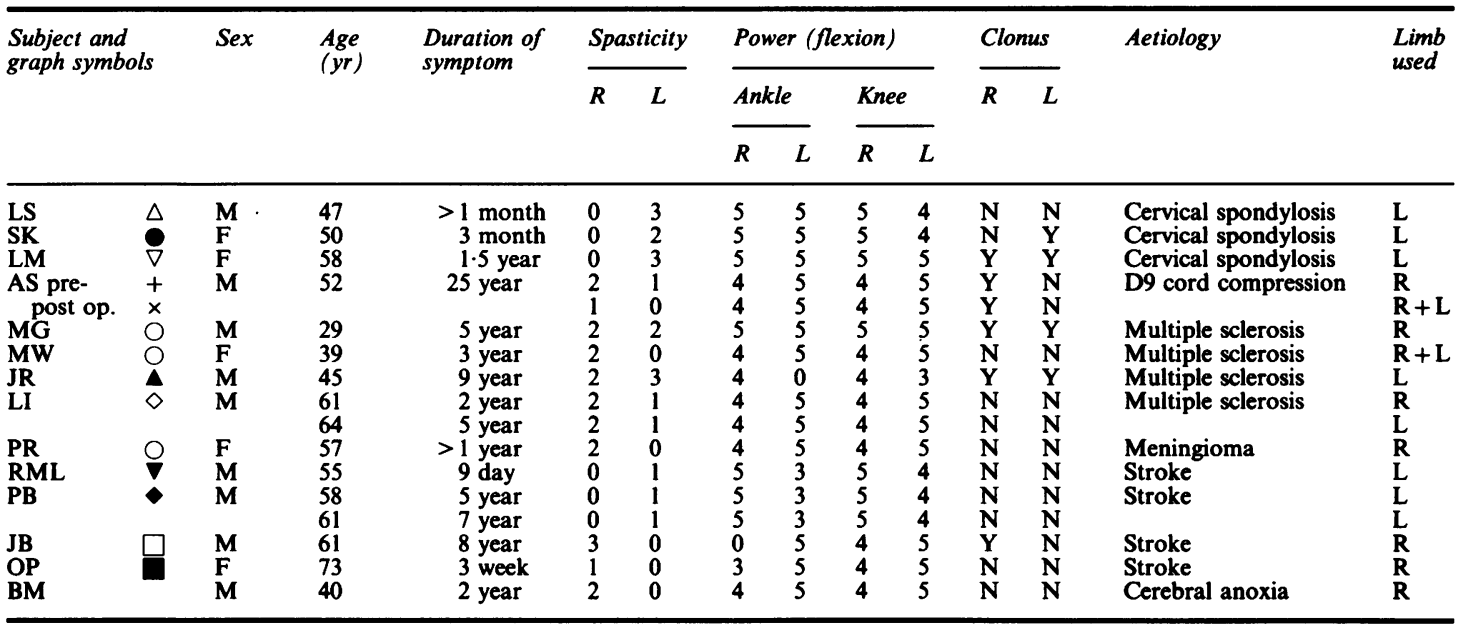


tion. For this purpose four older normal adult subjects (41 to 73 years) were recruited.

Test monosynaptic reflexes ( $\mathrm{H}$ reflexes) were elicited in the soleus muscle by electrical stimulation of the tibial nerve at frequencies close to $0 \cdot 2 \mathrm{~Hz}$. Except when reflex amplitude was explicitly studied the test reflexes were sub-maximal. The subjects were studied both at rest and during voluntary isometric contractions of soleus at predetermined levels of plantar flexion torque. Ankle and knee angles were maintained at $100^{\circ}$ and $120^{\circ}$ respectively. The reflex was recorded with surface EMG electrodes over soleus.

Presynaptic inhibition was studied by conditioning the reflex. Conditioning was obtained by maintained vibration of gastrocnemius-soleus, tibialis anterior, quadriceps or biceps femoris muscles using a physiotherapy vibrator $(100 \mathrm{~Hz}, 0.5-1 \mathrm{~mm}$ peak to peak amplitude) placed on the muscle belly. A series of 16 test reflexes was rectified, averaged and integrated; followed by 16 conditioned refiexes. The effect of the conditioning stimulus is expressed in the form conditioned reflex amplitude as a percentage of the test reflex $(\mathrm{C} / \mathrm{T} \%)$.

In some spastic subjects it was not possible to make a complete investigation. This was usually because muscle vibration (particularly of tibialis anterior, though the stimulus could be cutaneous rather than proprioceptive ${ }^{7}$ ), set off clonus in soleus. Sometimes this could be prevented by small adjustments of ankle angle about the standard $100^{\circ}$ position, by applying a brief plantar flexing force after each reflex, or by applying the vibrator for only a second or two before each reflex was elicited. In some subjects soleus could not be studied at rest because vibration consistently set up a tonic vibration reflex (TVR). We discarded all data where a TVR was observed.

\section{Results}

\section{Reflexes conditioned by muscle vibration}

(1) Normal subjects and spastic subjects indistinguishable from normal In normal subjects, autogenetic presynaptic inhibition of the soleus $\mathrm{H}$ reflex, produced by vibration of gastrocnemius-soleus, declines slightly during progressively stronger voluntary plantar flexions. This is illustrated in fig $1 \mathrm{~b}$. On the ordinate the conditioning action of vibration is expressed in the form conditioned reflex amplitude as a percentage of the control $(\mathrm{C} / \mathrm{T} \%$, zero is maximum inhibition, $100 \%$ is no inhibition). Plantar flexion torque is plotted along the abscissa. Inhibition produced by vibration of the antagonist muscle, tibialis anterior, is stronger, but declines more markedly with plantar flexion (fig la).

Two spastic subjects, MG and MW, showed inhibitory actions indistinguishable from the normal group. These patients had moderate spasticity but little weakness (resulting from multiple sclerosis in both cases). A third slightly spastic subject with a cerebral lesion also had a normal level of inhibition (BM, see fig 4).

All other spastic subjects provided results that were
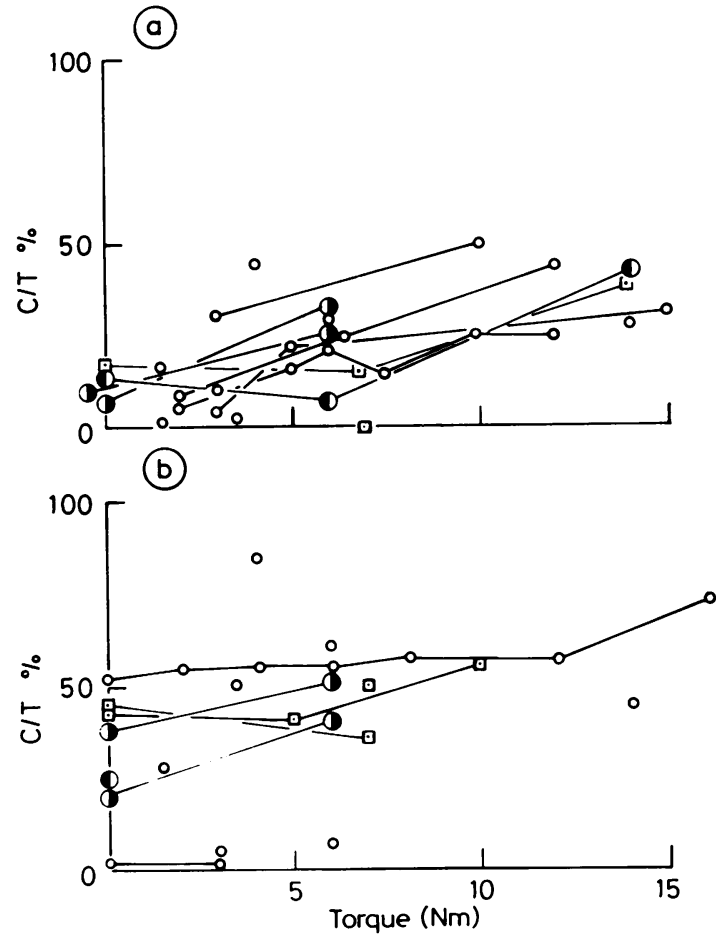

Fig 1 The effects of vibration of tibialis anterior (a) and gastrocnemius-soleus (b) on the soleus $\mathrm{H}$ reflex at different levels of voluntary plantar flexion. Ordinate: conditioned reflex as a percentage of test reflex amplitude (C/T\%). Abscissa: plantar flexion torque. $\bigcirc=$ young adult normal subjects. $\square=$ older adult normal subjects. For other symbols see table (both right and left legs were studied for subject $M W$ ).

different from normal. For ease of description they have been divided into two further groups. However, there is probably a continuous range from apparently normal to grossly altered and the whole group has been used for statistical analysis (see below).

(2) Spastic subjects with weak inhibition, reduced during contraction In these three subjects the strength of inhibition was less than normal but declined during plantar flexion, just as in the control group. This was most clearly seen when vibration of tibialis anterior was used as the conditioning stimulus. These effects are illustrated in fig 2 . In this figure (and in fig 3) the cross hatched areas indicate ranges of inhibitory strength that include more than $90 \%$ of the data points from normal subjects. The subjects showing these modest changes in presynaptic inhibition had slight to moderate spasticity and none had severe weakness. 


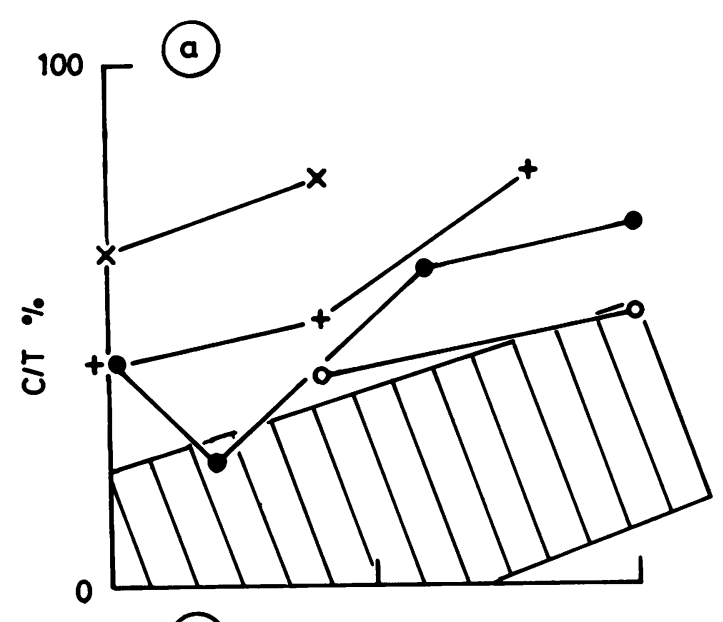

(b)

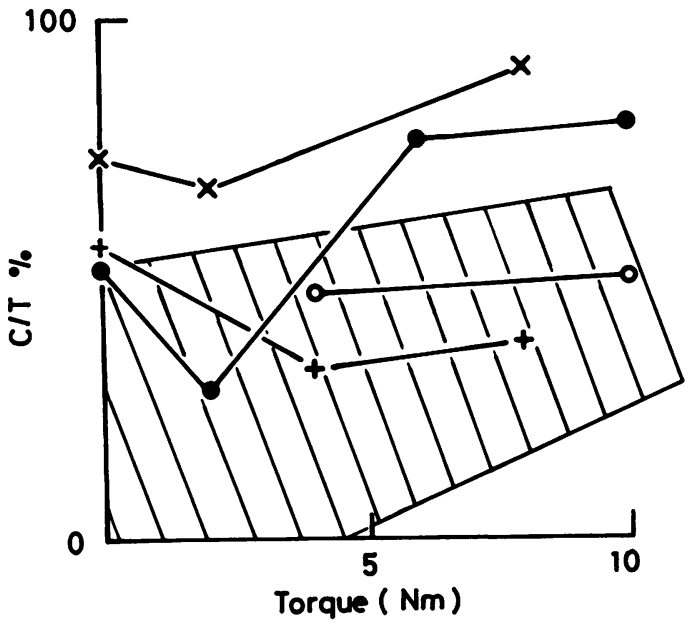

Fig 2 The effects of vibrating tibialis anterior (a) and gastrocnemius-soleus (b) on the soleus H reflex of three spastic subjects. Note that the right leg of subject AS was examined both before and after a decompressive laminectomy.

(3) Spastic subjects with very weak inhibition, either unchanged or increased during contraction These nine spastic subjects were furthest removed from the control group, in that the strength of inhibition at rest, or during weak soleus contractions, was very low. In some cases inhibition increased slightly during stronger voluntary plantar flexions (fig 3 ).

This group included all five subjects with severe weakness of ankle dorsiflexion and all four with severe spasticity. One subject (AS) appears in this group after having had a decompressive cervical laminectomy; data from before the operation are included
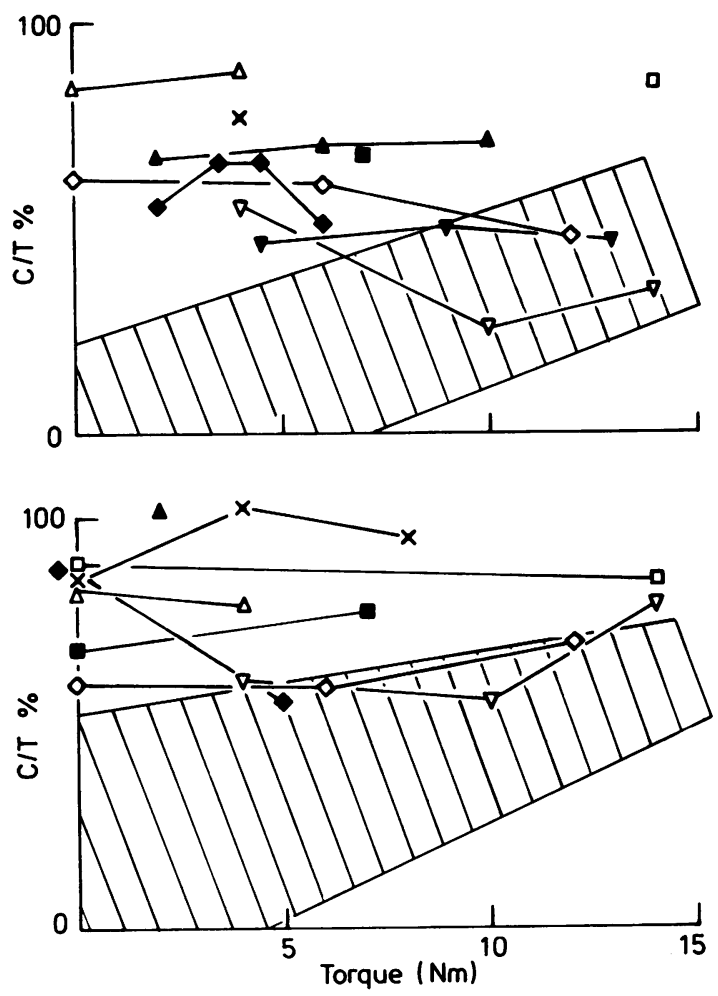

Fig 3 The effects of vibrating tibialis anterior and gastrocnemius-soleus on the soleus $\mathrm{H}$ reflex of nine spastic subjects. Note that the data for subject AS are for the left leg after a decompressive laminectomy.

in the previous group. His condition was clinically little changed. In subject $L I$ we investigated the effects of vibration of other muscles. Vibration of quadriceps produced no inhibition. Vibration of biceps femoris produced very little in comparison with normal subjects. $^{5}$

\section{Statistical analysis}

The Mann-Whitney $U$ test ${ }^{12}$ was applied to the estimates of inhibition for the set of spastic subjects compared to the sample of control subjects. Because inhibition was related to voluntary torque the data was divided into six sets of pairs in three ranges of torque $(0<$ low torque $\leqslant 5 \mathrm{Nm}, 5<$ medium torque $\leqslant 10 \mathrm{Nm}$ and $10<$ high torque $\leqslant 15 \mathrm{Nm}$; each for both soleus and tibialis anterior conditioning vibration). Data obtained at rest were not used because this could include postsynaptic inhibition.

Inhibition produced by tibialis anterior vibration was weaker in spastic subjects compared with normal ones at all three torque levels $(\mathrm{p}<0.002, \mathrm{p}<0.01$, 


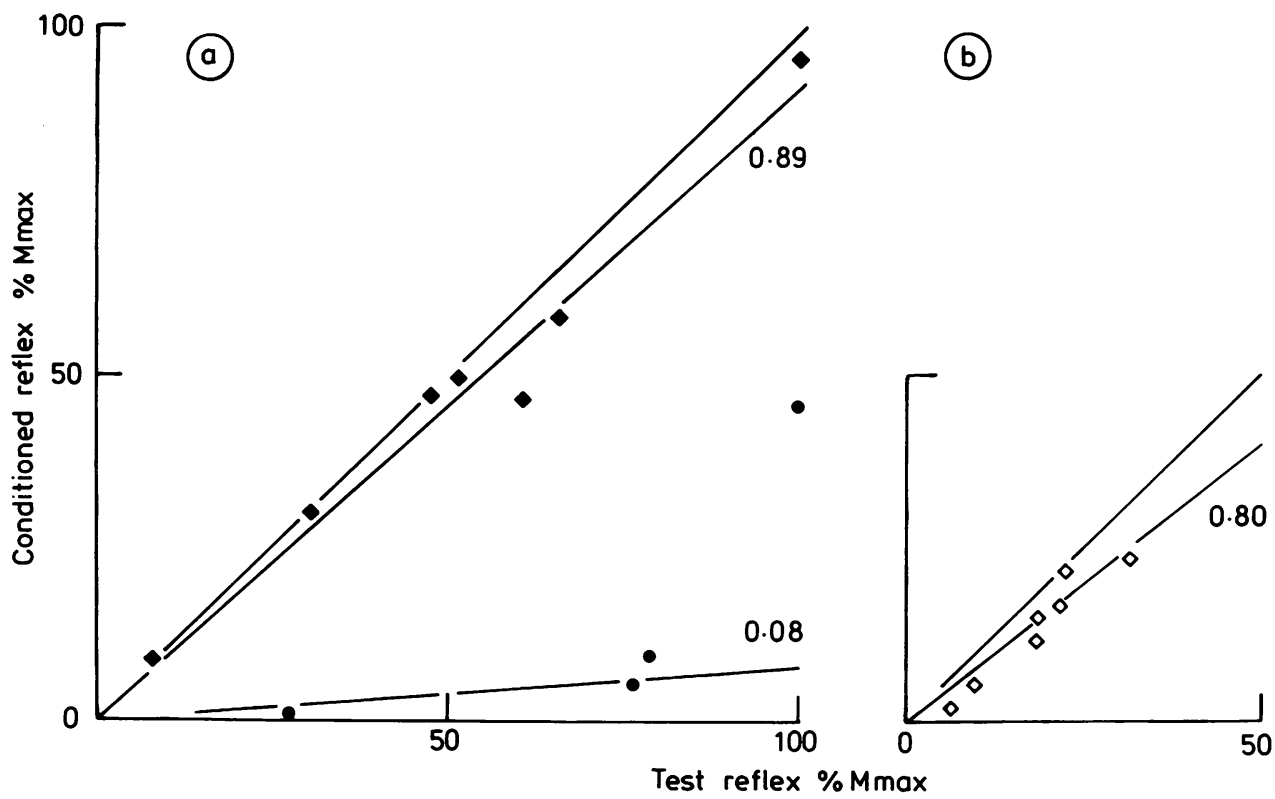

Fig 4 Estimates of presynaptic inhibition of the soleus $\mathrm{H}$ reflex from vibration of gastrocnemius-soleus in three spastic subjects at rest. The test reflex amplitude was deliberately varied over the widest possible range by adjustment of the strength of the test stimulus to the tibial nerve. The amplitude of the conditioned reflex (expressed as a percentage of the maximal direct muscle response recorded in soleus with supramaximal stimulation) has been plotted (ordinate) against test reflex amplitude (abscissa). The usual estimates of inhibition $(C /)$ are given by the regression lines. (a) subjects $P B(\bullet)$ and $B M(-)$ : the regression lines have been fitted through the origin and all data points except those for the largest reflexes. Note that subject $B M$ has a normal level of inhibition, in $P B$ it is much reduced. Substantial inhibition was still present when $B M$ made a small plantar flexion. (b) subject $L I(\diamond)$ : the regression line has been fitted through the origin and all of the data points.

$\mathrm{p}=0.12$ for low, medium and high torques respectively; two tailed tests). In the case of vibration of soleus itself inhibition was also weaker in spastic subjects but not significantly $(\mathrm{p}=0 \cdot 1, \mathrm{p}=0 \cdot 2, \mathrm{p}=$ $0 \cdot 2$ ).

The relationship between strength of inhibition and severity of symptoms within the spastic group was analysed by the Spearmann rank correlation coefficient. ${ }^{12}$ The data set for inhibition resulting from tibialis anterior vibration at medium torque were used because this included most subjects (only LS, JB and BM were absent). Presynaptic inhibition of the soleus reflex was significantly associated with the power of ankle dorsiflexion $\left(r_{s}=0.52\right.$ corrected for ties, $p=0.04$, one tailed test). There was no significant association with the clinical assessment of spasticity.

\section{Test reflex amplitude and strength of inhibition}

A retrospective analysis of the data described above did not suggest that the estimates of inhibition were related to reflex amplitude: subject LM showed a clear increase in test reflex amplitude with soleus contraction accompanied by a decrease in inhibition, just as in the control group; subjects LI, JR, PB and JB also gave larger test reflexes during contraction but their inhibition remained constant or increased.

For a more direct examination experiments were performed in which the amplitude of the test reflex was changed by controlling the size of the stimulus to the tibial nerve. The test stimulus was adjusted to produce the largest possible range of reflex amplitudes, which were expressed relative to the maximal direct muscle response recorded from soleus during supramaximal stimulation of the tibial nerve. The reflexes were conditioned by soleus vibration. Plots of conditioned versus test reflex amplitude are illustrated in fig 4. Subjects BM and PB both produced test reflexes up to maximal amplitude. For subject $P B$ the effect of soleus vibration was very small for all sizes of reflex. For subject BM inhibition was powerful but the estimate of it was smaller with the largest test reflex. Subject LI only produced small reflexes, all of which were weakly inhibited. 


\section{Discussion}

The strength of presynaptic inhibition in spastic subjects The experiments using soleus vibration as conditioning stimulus suggest, in agreement with earlier reports, that autogenetic inhibition of soleus Ia afferents is reduced in many subjects with upper motoneuron lesions and spasticity. Furthermore, there appears to be a relationship between loss of inhibition and the severity of weakness (cf. ref. 13 though this data obtained at rest could include postsynaptic actions). In this respect it should be remembered that our experimental group of subjects was selected for retention of some voluntary control of plantar flexion and thus was representative of only the less severe end of the clinical range. There was no clear correlation with aetiology. The reduction in inhibition involved both the autogenetic pathway and also actions from other muscles (cf. ref. 14 though their data could include postsynaptic actions). This suggests that the pathways from various muscles may converge at an interneuronal level before producing presynaptic inhibition (as in the cat, ${ }^{15}$ although autogenetic actions seem to be very weak in this species). The fact that inhibition was weak in comparison with controls even during soleus contraction strongly suggests reduction in a presynaptic component of inhibition. The reduced significance of the difference between spastic and control groups found at high torques would be anticipated because presynaptic inhibition is reduced during contraction in normal subjects. The behaviour of spastic and control groups converges during strong voluntary contraction. Changes in postsynaptic components could contribute to effects seen at rest.

A number of objections to this interpretation can be discounted. First, since the reduction in inhibition compared with control subjects was seen both at rest and during weak voluntary plantar flexion, it is unlikely that an inability to fully relax the soleus muscle fully leads to an incorrect overall conclusion. In normal subjects, presynaptic inhibition of soleus Ia afferents declines during contraction and we have concluded that descending control is mainly responsible for this inverse relation between presynaptic inhibition and plantar flexion torque. ${ }^{5}$ If this is correct, strength of inhibition may be most closely related to voluntary effort. The reduced inhibition seen during plantar flexion in weak spastic subjects might, then, simply result from an increased effort made to achieve the same torque. This is unlikely, however, to introduce a significant distortion because most spastic subjects showed reduced inhibition during small contractions, and not all were weak. We were unable to ask the subjects to perform contractions in both lower limbs with matched efforts and to measure torque on the contralateral side because many had bilateral deficits (even with unilateral lesions: cf. ref. 16). For the same reason we were not able to use the contralateral side as a control.

Second, the differences between spastic and normal subjects could result from reduced sensitivity of muscle spindles to vibration. There is no evidence for reduced spindle sensitivity, ${ }^{17}$ and tonic vibration reflexes could often be elicited at rest. This objection is also countered by the reduced inhibition that has been seen when electrical stimulation of the common peroneal nerve is used to condition the reflex. ${ }^{18}$

Third, the differences could involve postsynaptic actions. However, presynaptic components of inhibition should have been favoured by the fact that we performed the experiments during soleus contraction (see above). In normal subjects presynaptic inhibition of soleus reflexes can be produced by vibration of most muscles in the lower limb. ${ }^{5}$ All these pathways were diminished in action in spastic subjects. This total pattern of change again suggests that reduced presynaptic inhibition rather than changes in postsynaptic actions is responsible.

A fourth possible objection is that the estimate of inhibition from vibration is related to the size of the test reflex which differs between spastic and normal subjects. A reduction of inhibition was found with one of the weakly spastic subjects in the present group (subject BM, fig 4) when the test H-reflex involved discharge of a high proportion of the motoneuron pool. However, inhibition in this subject still fell within the normal range for all amplitudes of test reflex. In two other spastic subjects the inhibition was below normal for all amplitudes of test reflex. Although reflex size did not in practice alter the conclusions drawn from experiments in these three cases it is clear that the large reflexes often found in spastic subjects ${ }^{9}$ could lead to falsely low estimates of presynaptic inhibitory action.

A final objection is that the spastic group were older than many of the controls and that presynaptic inhibition declines with age. ${ }^{2}$ In our small group, studied both at rest and during contraction, there was no clear effect of age and all but three spastic subjects had less inhibition than similarly aged controls.

Overall, a reduction in the strength of presynaptic inhibition in subjects with upper motoneuron disease does appear to be the simplest explanation for the results described here.

\section{Modulation of presynaptic inhibition during contraction} in spastic subjects

Some spastic subjects exhibited presynaptic inhibition that reduced during active plantar flexion, just as in controls. More of them showed no change, or even a slight increase. Variation was also found by Pierrot- 
Deseilligny and Lacert ${ }^{16}$ when investigating the soleus $H$ reflex depression that occurs in normal subjects before contraction of the antagonist. This depression results in part from enhanced presynaptic inhibition. The phenomenon was absent in spastic subjects with lesions of the cerebral hemispheres, but present in subjects with sub-cortical lesions. It is possibly significant that all of our cerebral stroke patients came in the category which showed no decrease of inhibition during soleus contraction.

A slight increase in presynaptic inhibition, which was observed in some subjects, could, in the absence of descending control, result from spatial summation of group I afferents activated by the isometric contraction with the conditioning input (cf. ref. 19).

The mechanism of control of Ia presynaptic inhibition in normal and spastic subjects

In two thirds of our spastic subjects, the decline in soleus Ia presynaptic inhibition during contraction, which is characteristic of the normal situation, was absent. A simple explanation for this phenomenon would be damage to a descending system which inhibits the spinal pathway of presynaptic inhibition. In the cat, stimulation of the contralateral red nucleus or pyramidal tract, or ipsilateral reticular formation all inhibit Ia presynaptic inhibition. ${ }^{20}$ Two descending pathways are particularly important for this effect: the rubrospinal tract and the ventral reticulospinal system. ${ }^{21}$ In the case of the rubrospinal tract, the inhibition of presynaptic inhibition is known to converge with peripheral, flexor reflex afferent actions $^{22}$ on the first order interneuron in the trisynaptic pathway of presynaptic inhibition. ${ }^{20}$ In contrast, the ventral reticulospinal system operates independently on the last order interneuron. We have obtained some evidence for actions from flexor reflex afferents on Ia presynaptic inhibition in man but there is no indication as to which level of descending control might be most important in human voluntary isometric contractions; (it should be noted that the rubrospinal tract may be rudimentary in $\operatorname{man}^{23}$ ).

If the only descending controls were inhibitory we would expect the consequence of lesions to these systems to be a release of presynaptic inhibition at the segmental level. This appears to be the situation in the acutely spinal man ${ }^{24} 25$ (though changes in postsynaptic actions could be present in these experiments). However, the long term condition in most spastic subjects, with cerebral or partial spinal lesions, is reduced inhibition. This could result from damage to pathways that facilitate presynaptic inhibition. In the cat, stimulation of the median longitudinal fasciculus, medial and descending vestibular nuclei, contralateral fastigial nucleus and, rarely, sensorimotor cortex all produce primary afferent depolarisation in flexor and extensor Ia afferents. ${ }^{26}$ Although some of these actions could partly involve inhibition of the previously mentioned descending inhibitory pathways, there are clearly several candidates for excitatory control. It is likely that the distributed nature of the descending controls of presynaptic inhibition explains the rather variable reactions of spastic subjects in this respect, particularly where the lesions are subcortical.

Contribution of reduced Ia presynaptic inhibition to spasticity

Loss of this component of inhibition could make some contribution to the exaggerated stretch reflexes of lower limb extensor muscles in spasticity. However, three of our subjects showing spasticity appeared to have a normal level of inhibition and control, suggesting that other factors play an important part. Furthermore, there was no significant (inverse) correlation between spasticity and strength of presynaptic inhibition in our spastic group.

Loss of the powerful presynaptic inhibition directed from flexor muscles to extensors could contribute to the reduced reciprocal innervation which characterises spasticity. ${ }^{7}$ This would be manifest in the lower limb as a lack of suppression of extensor stretch reflexes during voluntary flexion movement. Such a conclusion is supported by the significant correlation we have found between presynaptic inhibition and strength of ankle dorsiflexion in the spastic subjects. Any reduction in postsynaptic reciprocal inhibition would also contribute to reduced reciprocal innervation. There is evidence from the upper limb that stretch reflexes in spastic antagonists may indeed impair fast voluntary movements. ${ }^{27}$

We thank Professor WB Matthews and Drs C Davis, RCD Greenhall, N Hyman and J Oxbury for allowing us to perform the investigations on their patients.

\section{References}

${ }^{1}$ Lance JW, DeGail P, Neilson PD. Tonic and phasic spinal cord mechanisms in man. $J$ Neurol Neurosurg Psychiatry 1966;29:535-44.

${ }^{2}$ Delwaide PJ. Etude Experimentale de l'Hyperreflexie Tendineuse en Clinique Neurologique 1971; Bruxelles: Editions Arscia.

${ }^{3}$ Ashby P, Verrier M. Neurophysiological changes in hemiplegia: possible explanation for the initial disparity between muscle tone and tendon reflexes. Neurology (Minneap) 1976;26:1145-51.

${ }^{4}$ Somerville J, Ashby P. Hemiplegic spasticity: neurophysiological studies. Arch Phys Med Rehab 1978; 59:592-6.

${ }^{5}$ Iles JF, Roberts RC. Presynaptic inhibition of mono- 
synaptic reflexes from human soleus muscle. $J$ Physiol (Lond) 1981;317:59P.

${ }^{6}$ Basmajian JV, Szatmari A. Effect of Largactil (chlorpromazine) on human spasticity and electromyogram. AMA Archives of Neurology and Psychiatry 1955;73:224-31.

${ }^{7}$ Dimitrijevic MR, Nathan PW. Studies of spasticity in man. 1. Some features of spasticity. Brain 1967;90:1-28.

${ }^{8}$ Mazieres L. L'effet d'une stimulation conditionnante depend de l'amplitude du reflexe monosynaptique test. Etude chez l'homme et chez chat. 1983; These. L'Universite Pierre et Marie Curie, Paris 6.

${ }^{9}$ Taylor S, Ashby P, Verrier M. Neurophysiological changes following traumatic spinal lesions in man. $J$ Neurol Neurosurg Psychiatry 1984;47:1102-8.

${ }^{10}$ Iles JF, Roberts RC. Presynaptic inhibition of monosynaptic reflexes from soleus muscle in spastic human subjects. J Physiol (Lond) 1983;341:29-30P.

${ }^{11}$ Read DJ, Matthews WB, Higson RH. The effect of spinal cord stimulation on function in patients with multiple sclerosis. Brain 1980;103:803-33.

${ }^{12}$ Siegel S. Nonparametric Statistics for the Behavioral Sciences. 1956; New York: McGraw Hill.

${ }^{13}$ Delwaide PJ. Human monosynaptic reflexes and presynaptic inhibition. In: Desmedt JE, ed. New Developments in Electromyography and clinical Neurophysiology. Basel:Karger, 1973:508-22.

${ }^{14}$ Burke D, Ashby P. Are spinal "presynaptic" inhibitory mechanisms suppressed in spasticity? $J$ Neurol Sci 1972;15:321-6.

${ }^{15}$ Brink E, Jankowska E, Skoog B. Convergence on to interneurons subserving primary afferent depolarisation of group I afferents. $J$ Neurophysiol 1984;51:432-49.

${ }^{16}$ Pierrot-Deseilligny E, Lacert P. Amplitude and variability of monosynaptic reflexes prior to various voluntary movements in normal and spastic man. In: Desmedt JE, ed. New Developments in Electromyography and Clinical Neurophysiology, vol. 3. Basel: S Karger 1973:538-49.

${ }^{17}$ Burke D. A reassessment of the muscle spindle con- tribution to muscle tone in normal and spastic man. In: Feldman RG, Young RR, Koella WP, eds. Spasticity: Disordered Motor Control. Chicago: Year Book Medical Publishers, 1980:261-78.

${ }^{18}$ El-Tohamy A, Sedgwick EM. Spinal inhibitory mechanisms in spasticity. Electroencephalogr Clin Neurophysiol 1982;53:3-4P.

${ }^{19}$ Devanandan MS, Eccles RM, Stenhouse D. Presynaptic inhibition evoked by muscle contraction. J Physiol (Lond) 1966;185:471-85.

${ }^{20}$ Rudomin P, Jiminez I, Solodkin M, Duenas S. Sites of action of segmental and descending control of transmission on pathways mediating PAD of $\mathrm{Ia}$ and $\mathrm{Ib}$ afferent fibres in cat spinal cord. $J$ Neurophysiol 1983;50:743-69.

${ }^{21}$ Lundberg A. Inhibitory control from the brain stem of transmission from primary afferents to motoneurons, primary afferent terminals and ascending pathways. In: Sjolund B, Bjorklund A, eds. Brain Stem Control of Spinal Mechanisms. Amsterdam: Elsevier, 1982: 179-224.

${ }^{22}$ Hongo T, Jankowska E, Lundberg A. The rubrospinal tract. III. Effects on primary afferent terminals. Exp Brain Res 1972;15:39-53.

${ }^{23}$ Nathan PW, Smith MC. The rubrospinal and central tegmental tracts in man. Brain 1982;105:223-69.

${ }^{24}$ Ashby P, Verrier M, Lightfoot E. Segmental reflex pathways in spinal shock and spinal spasticity in man. J Neurol Neurosurg Psychiatry 1974;37:1352-60.

${ }^{25}$ Ashby P, Verrier M. Neurophysiological changes following spinal cord lesions in man. Can $J$ Neurol $S c i$ 1975;2:91-100.

${ }^{26}$ Baldissera F, Hultborn H, Illert M. Integration in spinal neuronal systems. In: Brooks VB, ed. Handbook of Physiology, Section 1 The Nervous System, volume II Motor Control, part I. Bethesda MD: American Physiological Society, 1981:509-95.

${ }^{27}$ Mizhari E, Angel RW. Impairment of voluntary movement by spasticity. Ann Neurol 1979;5:594. 